\title{
Continuous-flow sheathless diamagnetic particle separation
}

\section{in ferrofluids}

Yilong Zhou ${ }^{a}$, Le Song ${ }^{\mathrm{b}}$, Liandong Yu ${ }^{\mathrm{b}, *}$, Xiangchun Xuan ${ }^{\mathrm{a}, *}$

${ }^{a}$ Department of Mechanical Engineering, Clemson University, Clemson, SC 29634-0921, USA

${ }^{b}$ School of Instrument Science and Opto-electronic Engineering, Hefei University of Technology, Hefei 230009, China

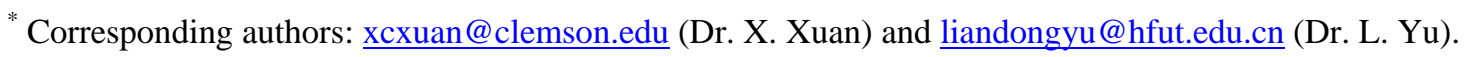




\begin{abstract}
Separating particles from a complex mixture is often necessary in many chemical and biomedical applications. This work presents a continuous-flow sheathless diamagnetic particle separation in ferrofluids through U-shaped microchannels. Due to the action of a size-dependent magnetic force, diamagnetic particles are focused into a single stream in the inlet branch of the U-turn and then continuously separated into two streams in its outlet branch. A 3D numerical model is developed to predict and understand the diamagnetic particle transport during this separation process. The numerical predictions are found to agree well with the experimental observations in a systematic study of the effects of multiple parameters including ferrofluid flow rate, concentration and magnet-channel distance. Additional numerical studies of the geometric effects of the U-turn reveal that increasing the outlet-branch width of the U-turn can significantly enhance the diamagnetic particle separation in ferrofluids.
\end{abstract}

Keywords: Ferrofluid, diamagnetic particle separation, magnetophoresis, microfluidics. 


\section{Introduction}

Separating particles (either synthetic or biological) from a binary or more complex mixture is often necessary in many chemical and biomedical applications [1-4]. Continuous-flow separation of particles based on intrinsic properties (e.g., size and shape) has been demonstrated in microfluidic devices by the use of a variety of force fields (either externally applied or internally induced) including acoustic [5,6], electric [7-9], hydrodynamic [10-12], magnetic [13-15], and optical [16,17] forces etc. [18-20]. Among these methods, magnetic field-driven particle separation exhibits several advantageous features over the others such as low cost and fluidheating free (if permanent magnets are used) as well as insensitivity to fluid properties (e.g., $\mathrm{pH}$ ) [21-24]. This type of separation has been implemented in primarily two different modes: one is to separate magnetic (or magnetically tagged) particles from diamagnetic (or less magnetic) particles based on their difference in magnetic susceptibility via positive magnetophoresis induced in a diamagnetic fluid [25-32], and the other is to separate diamagnetic particles from diamagnetic particles based on their difference in size or other non-magnetic properties (e.g., shape) via negative magnetophoresis induced in a magnetic fluid [33-40].

Diamagnetic particle separation has thus far been demonstrated in either paramagnetic salt solutions (e.g., $\mathrm{MnCl} 2$ and $\mathrm{GdCl} 3$ ) [41,42] or ferrofluids [43-45]. Paramagnetic solutions usually have a weak magnetic susceptibility unless the concentration of the paramagnetic salt goes high (which, however, may cause severe adverse effects on the sample) [46]. Therefore, superconducting magnets sometimes need to be used in order to achieve a high-throughput manipulation of diamagnetic particles in paramagnetic solutions [41]. In contrast, ferrofluids are opaque colloidal suspensions of superparamagnetic nanoparticles and have a magnetic 
susceptibility several orders of magnitude larger than that of paramagnetic solutions [47]. Therefore, regular permanent magnets and even electric magnets are normally sufficient for manipulating diamagnetic particles in ferrofluids with a decent flow throughput [48-54]. However, the majority of the reported diamagnetic particle separations in magnetic fluids need to use a sheath fluid, which is also magnetic and typically the pure suspending fluid of the particle suspension, to pre-focus the particle mixture [34-37,40-43]. This not only complicates the flow control and device fabrication but also dilutes the separated particles at the cost of the magnetic fluid.

Our group has recently developed two sheath-free approaches to continuous-flow diamagnetic particle separation in ferrofluids. In the first approach, two permanent magnets are placed offset to each other along the length of a straight microchannel with dissimilar magnet-channel distances, where the first magnet is to focus the particle mixture into a single stream and the second magnet is to deflect particles to size-dependent flow paths for a continuous separation [55]. The experimental observations are found to agree quantitatively with the predictions of an analytical model [55]. In the second approach, a single permanent magnet is placed near a Ushaped microchannel, where particles are magnetically focused without a sheath flow in the inlet-branch and continuously separated into two streams in the outlet-branch via size-dependent magnetophoresis [56]. However, due to the use of an extremely low-concentration ferrofluid ( $0.012 \%$ volume), polystyrene particles were observed to behave like "magnetic" particles in our experiment [56] due to perhaps the attachment of magnetic nanoparticles onto their surfaces [57]. 
This work is aimed to demonstrate a true diamagnetic particle separation in a U-shaped microchannel by re-suspending particles into a slightly diluted EMG 408 ferrofluid (Ferrotec Corp.) with a concentration of around $0.6 \%$ (volume). More importantly, a 3D numerical model is developed to predict and understand the transport of diamagnetic particles during their separation in ferrofluid microflows. The experiment and simulation are combined to perform a comprehensive parametric study of the effects of multiple parameters on the diamagnetic particle separation in the U-shaped microchannel. In addition, the validated numerical model is used to further investigate the geometric effects of the U-turn with the goal of optimizing the design and control of U-shaped microchannels for the best separation performance.

\section{Experiment}

\subsection{Microfluidic chip fabrication}

Fig. 1 shows a picture of the microfluidic chip used in experiments. It was fabricated with polydimethylsiloxane (PDMS) using a custom-modified soft lithography method as detailed elsewhere [58]. The U-shaped microchannel is uniformly $200 \mu \mathrm{m}$ wide and $40 \mu \mathrm{m}$ deep. It consists of a $6 \mathrm{~mm}$ long straight section at each end (i.e., inlet and outlet) and a U-turn in the middle. The two branches of the U-turn (named as inlet branch and outlet branch hereafter) are each $6 \mathrm{~mm}$ long and separated with a gap of $800 \mu \mathrm{m}$. A neodymium-iron-boron permanent magnet $\left(B 222,1 / 8^{\prime \prime} \times 1 / 8^{\prime \prime} \times 1 / 16^{\prime \prime}, \mathrm{K} \& \mathrm{~J}\right.$ Magnets, Inc.) is embedded into the PDMS slab and in direct contact with the glass slide. It has an edge-to-edge distance of $550 \mu \mathrm{m}$ from the inlet straight section and $800 \mu \mathrm{m}$ (note: this value was varied in the experiment to study the effects of magnet-channel distance on particle separation) from the inlet branch of the U-turn. Its 
magnetization direction is through the $1 / 16^{\prime \prime}$ thickness and perpendicular to the two branches of the U turn.

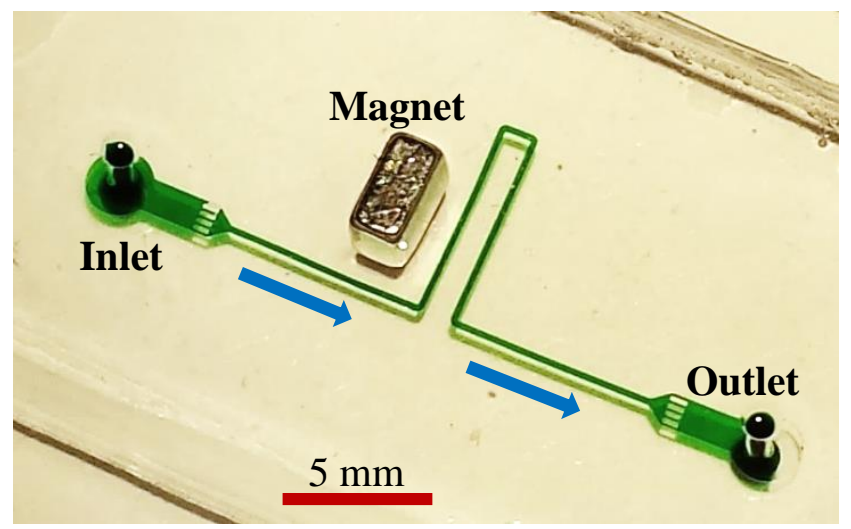

Fig. 1: Picture of the microfluidic chip (the microchannel and reservoirs are filled with green food dye for clarity) used in experiments. The permanent magnet is embedded into the PDMS slab and placed nearby the inlet branch of the U-shaped microchannel. The block arrows indicate the ferrofluid flow directions in particle separation experiments.

\subsection{Particle solution preparation and experimental method}

The particle solution was prepared by mixing and re-suspending $5 \mu \mathrm{m}$ - and $15 \mu \mathrm{m}$-diameter diamagnetic polystyrene particles (both with a coefficient of variation smaller than $2 \%$, Sigma Aldrich) in $0.5 \times$ EMG 408 ferrofluid (Ferrotec Corp.) to a final concentration of $10^{6}-10^{7}$ particles per milliliter. The dilution value was varied from $0.4 \times$ to $0.6 \times$ in the experiment to study the effects of ferrofluid concentration on particle separation. To reduce particle aggregations and adhesions to microchannel walls, 0.5\% (in volume) Tween 20 (Fisher Scientific) was added to the suspension. Prior to uses, the particle solution was stirred using a fixed speed vortex mixer (Fisher Scientific) for a uniform dispersion. It was driven to flow through the Ushaped microchannel by an infusion syringe pump (KD Scientific) via a polyether ether ketone (PEEK) tubing (IDEX Health \& Science) inserted into the inlet reservoir. The outlet reservoir was also connected to a tubing to export the solution out of the microchannel. Particle motion 
was visualized at different locations of the microchannel using an inverted microscope (Nikon Eclipse TE2000U, Nikon Instruments) and recorded with a CCD camera (Nikon DS-Qi1Mc) at a rate of 15 frames per second. The captured digital videos and images were post-processed using the Nikon imaging software (NIS-Elements AR 2.30).

\section{Simulation}

In order to predict and understand the diamagnetic particle separation in ferrofluid flows, a 3D numerical model was developed to simulate the magnetophoretic particle transport in the Ushaped microchannel. Similar to our earlier work [59], this model considers only the one-way actions that the flow field (via the drag force) and the magnetic field (via the magnetic force) have on the suspended particles. The re-actions of these particles on the flow and magnetic fields, as well as the dipole-dipole interactions between themselves, are, however, neglected considering the low particle concentration in our experiments [60,61]. Moreover, the influence of the ferrofluid concentration gradient, which results from the re-distribution of superparamagnetic nanoparticles in response to a magnetic field, is assumed small on the diamagnetic particle transport and separation. This assumption has been verified by a two-dimensional numerical model that considers the full coupling between the ferrofluid flow and concentration fields in the U-shaped microchannel (see the Appendix for details). It is found that the ferrofluid concentration in the bulk fluid remains almost unvaried (see Fig. A1 in the Appendix), and can thus be treated as uniform throughout the microchannel in our 3D model.

\subsection{Computational domain}


Fig. 2 shows the isometric view of the 3D computational domain, which covers the entire U-turn of the microchannel and part of the straight sections at the channel inlet and outlet (see labeled dimensions). Specifically, the straight channel section at the inlet is truncated to $3000 \mu \mathrm{m}$, which is sufficient to take account of the particle deflection and focusing therein due to magnetophoresis. The straight channel section at the outlet is cut to only $1000 \mu \mathrm{m}$ because our experiment and simulation both indicate no significant particle trajectory change occurs beyond this length. Structured meshes are used over the entire computational domain as viewed from the insets I, II, and III in Fig. 2. The grid size at the corner of the inlet branch (see inset I) selfadaptively increases from the inner wall to the outer wall. Moreover, the grid size is set to decrease from the microchannel center to each of the four walls at a constant ratio (see insets II and III). With this approach, no additional boundary layer meshes, which were employed in the 3D model in our earlier work [59] are needed because the meshes near channel walls are fine enough to trace the particle position accurately.

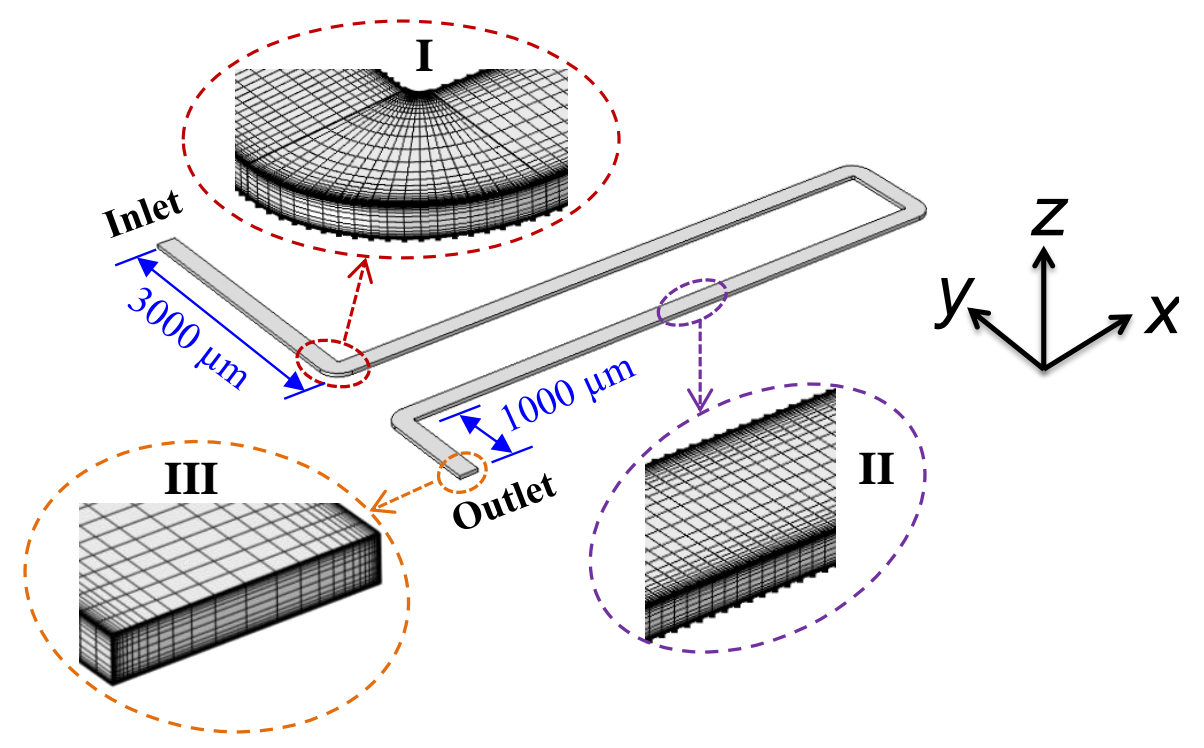

Fig. 2: Isometric view of the 3D computational domain of the U-shaped microchannel. Note that the lengths (labeled on the plot) of the straight sections at the channel inlet and outlet are both 
truncated to save computer time. The insets I, II and III show the enlarged views of the structured meshes at the corner of the inlet branch, the middle of the outlet branch and the channel outlet, respectively.

\subsection{Governing equations and boundary conditions}

The ferrofluid is viewed as a magnetic fluid of a uniform concentration, $c_{0}$, for which the steadystate flow field in the U-shaped microchannel, $\mathbf{u}$, is governed by the continuity and NavierStokes equations $[62,63]$,

$$
\begin{gathered}
\nabla \cdot \mathbf{u}=0 \\
\rho \mathbf{u} \cdot \nabla \mathbf{u}=-\nabla p+\eta \nabla^{2} \mathbf{u}+\mathbf{f}_{m}
\end{gathered}
$$

where $\rho, p$ and $\eta$ are the ferrofluid density, pressure and viscosity, respectively, and $\mathbf{f}_{m}$ is the magnetic body force given by [47],

$$
\begin{gathered}
\mathbf{f}_{m}=\mu_{0} \mathbf{M}_{f} \cdot \nabla \mathbf{H} \\
\mathbf{M}_{f}=c_{0} \mathbf{M}_{d}[\operatorname{coth}(\alpha)-1 / \alpha] \\
\alpha=\frac{\pi d^{3} \mu_{0} M_{d} H}{6 k_{B} T}
\end{gathered}
$$

In the above, $\mu_{0}$ is the permeability of the free space, $\mathbf{M}_{f}$ is the magnetization of the ferrofluid, $\mathbf{H}$ is the magnetic field with $H$ being the magnitude, $\mathbf{M}_{d}$ is the saturation moment of the superparamagnetic nanoparticle with $M_{d}$ being the magnitude, $d$ is the nominal diameter of the superparamagnetic nanoparticles in the ferrofluid, $k_{B}$ is the Boltzmann constant, and $T$ is the ferrofluid temperature. The inlet of the microchannel is imposed with the experimentally used volume flow rate, and the outlet is at zero pressure. A no-slip boundary condition is applied to all channel walls. 
To simulate and trace the diamagnetic particle trajectories in the separation process, we neglect the inertial effect and consider only the contributions of fluid flow, $\mathbf{u}$, negative magnetophoresis, $\mathbf{u}_{m}$, and gravity-buoyancy action, $\mathbf{u}_{g b}$, to the particle velocity, $\mathbf{u}_{p}$, i.e.,

$$
\begin{gathered}
\mathbf{u}_{p}=\mathbf{u}+\mathbf{u}_{m}+\mathbf{u}_{g b} \\
\mathbf{u}_{m}=-\frac{2 \mu_{0} a^{2} \mathbf{M}_{f} \cdot \nabla \mathbf{H}}{9 \eta f_{D}} \\
\mathbf{u}_{g b}=\frac{2 a^{2}\left(\rho_{p}-\rho\right)}{9 \eta f_{D}} \mathbf{g}
\end{gathered}
$$

In the above, $a$ and $\rho_{p}$ are the radius and mass density of diamagnetic particles, $f_{D}$ is the drag coefficient that accounts for the wall retardation effects, and $\mathbf{g}$ is the gravitational acceleration. Similar to our earlier work [49,59], different expressions of $f_{D}$ are used for particle motions parallel and normal to the channel walls, respectively, and skipped here for brevity. The magnetization of diamagnetic particles is assumed negligible as compared to that of the ferrofluid in Eq. (7). The magnetic field, $\mathbf{H}$, in Eqs. (3) and (7) are obtained using the analytical formulae derived by Furlani for rectangular permanent magnets [64], which, as stated in our earlier work [59], can greatly reduce the computer time due to the elimination of the necessary air box around the magnet [65].

\subsection{Numerical method}

The 3D numerical model was developed and solved in $\mathrm{COMSOL}^{\circledR} 4.4$ using the Palmetto Cluster at Clemson University. Briefly, the 3D magnetic field was determined directly from Furlani's analytical formulae for rectangular magnets [64] that were input to COMSOL as variables. The ferrofluid flow velocity, $\mathbf{u}$, was solved from Eq. (1) and Eq. (2) in the Laminar 
Flow module. The diamagnetic particle motion was traced using the intrinsic Streamline function in COMSOL via Eq. (6) in terms of the following velocity components in the three directions:

$$
\begin{gathered}
u_{p_{-} x}=u_{x}-\frac{2 \mu_{0} a^{2} M_{f}}{9 \eta f_{D} H}(\mathbf{H} \cdot \nabla) H_{x} \\
u_{p_{-} y}=u_{y}-\frac{2 \mu_{0} a^{2} M_{f}}{9 \eta f_{D} H}(\mathbf{H} \cdot \nabla) H_{y} \\
u_{p_{-} z}=u_{z}-\frac{2 \mu_{0} a^{2} M_{f}}{9 \eta f_{D} H}(\mathbf{H} \cdot \nabla) H_{z}-\frac{2 a^{2}\left(\rho_{p}-\rho\right) g}{9 \eta f_{D}}
\end{gathered}
$$

where $u_{i}(i=x, y, z)$ is the fluid velocity component, $M_{f}$ is the magnitude of ferrofluid magnetization in Eq. (4), and $H_{i}(i=x, y, z)$ is the magnetic field component that can be found from Ref. [64]. The maximum mesh size was set to $6 \mu \mathrm{m}$ according to a grid independence study.

Fig. 3 shows the isometric view of the magnetic field contour within the U-shaped microchannel, which indicates the decrease in magnetic field from the inlet branch to the outlet branch of the Uturn. The material properties involved in the simulation are summarized in Table 1.

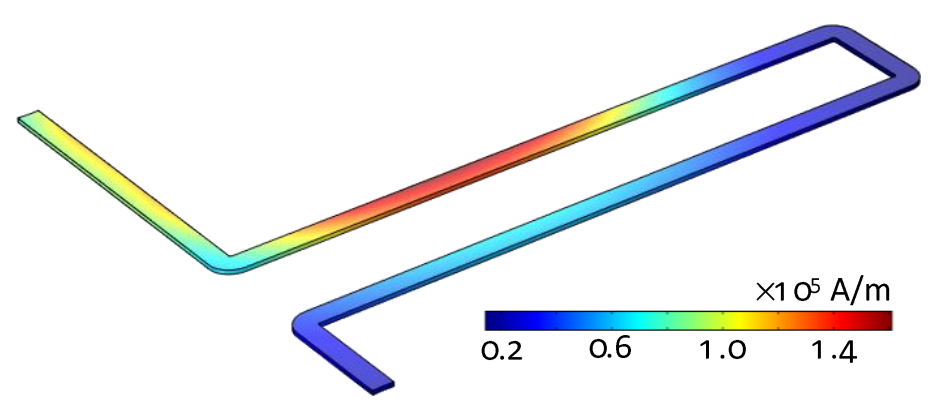

Fig. 3: Isometric view of the magnetic field contour within the U-shaped microchannel.

Table 1: Material properties used in the simulation.

\begin{tabular}{cll}
\hline Symbol & \multicolumn{1}{c}{ Description } & \multicolumn{1}{c}{ Value } \\
\hline$M_{s}$ & Residual magnetization of the magnet & $1.05 \times 10^{6} \mathrm{~A} / \mathrm{m}$ \\
$c_{0}$ & Ferrofluid concentration (0.5 $\times$ EMG 408) & $0.6 \%$ \\
$d$ & Diameter of magnetic nanoparticles & $10 \mathrm{~nm}$ \\
$M_{d}$ & Saturation moment of magnetic nanoparticles & $4.38 \times 10^{5} \mathrm{~A} / \mathrm{m}$ \\
$\eta$ & Dynamic viscosity of the ferrofluid & $1.05 \times 10^{-3} \mathrm{~kg} / \mathrm{ms}$ \\
\hline
\end{tabular}




\begin{tabular}{cll}
\hline$\rho$ & Mass density of the ferrofluid & $1070 \mathrm{~kg} / \mathrm{m}^{3}$ \\
$2 a$ & Diameter of diamagnetic particles & $5 \mu \mathrm{m}$ and $15 \mu \mathrm{m}$ \\
$\rho_{p}$ & Density of diamagnetic particles & $1050 \mathrm{~kg} / \mathrm{m}^{3}$ \\
\hline
\end{tabular}

\section{Results and discussion}

\subsection{Demonstration of diamagnetic particle separation}

Fig. 4 compares the experimentally obtained images and the numerically predicted trajectories of $5 \mu \mathrm{m}$ and $15 \mu \mathrm{m}$ particles in $0.5 \times$ EMG 408 ferrofluid through the U-shaped microchannel. Four observation windows I-IV (see also Fig. 5 for their locations) are selected to demonstrate the development of this diamagnetic particle separation. The permanent magnet is $800 \mu \mathrm{m}$ away from the inlet branch of the U-turn. The flow rate of the particle suspension is $450 \mu \mathrm{l} / \mathrm{h}$, which corresponds to an average velocity of $15.6 \mathrm{~mm} / \mathrm{s}$. The composite images are each a superposition of a sequence of top-view snapshot images. At the entrance region of the U-turn (window I), 15 $\mu \mathrm{m}$ particles are focused to a single stream along the lower wall of the inlet branch. In contrast, the magnetic deflection of $5 \mu \mathrm{m}$ particles is weaker and not completed until after they move out of window I. This happens because of the size-dependent magnetophoretic velocity, $\mathbf{u}_{m}$, in Eq. (7). On moving through window II at the inlet branch closest to the magnet, $5 \mu \mathrm{m}$ and $15 \mu \mathrm{m}$ particles migrate in a single tight stream near the lower wall of the inlet branch. Meanwhile, ferrofluid nanoparticles are accumulated onto the upper wall of the inlet branch due to positive magnetophoresis, which is explained in the Appendix. The focusing status of diamagnetic particles continues in window III where they move along the inner corner of the $\mathrm{U}$ turn. 

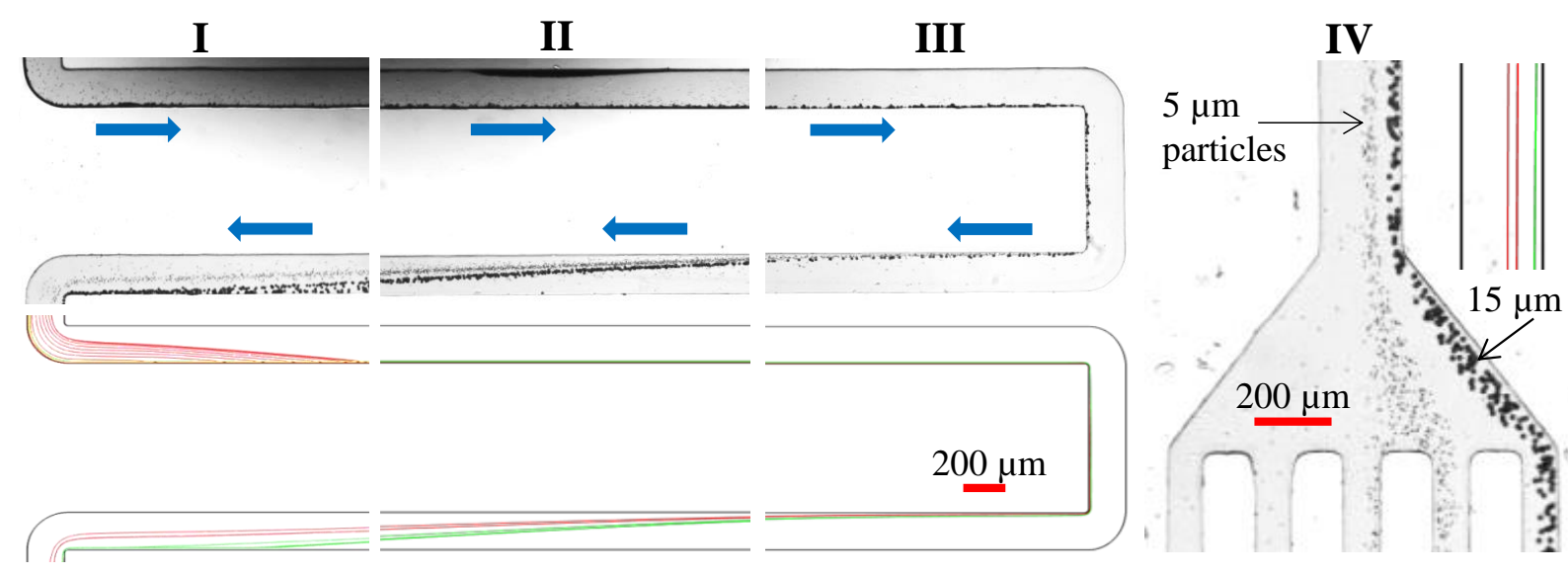

Fig. 4: Comparison of top-view experimental images and numerical predictions (red lines for 5 $\mu \mathrm{m}$ particles and green lines for $15 \mu \mathrm{m}$ particles) for diamagnetic particle separation in $0.5 \times$ EMG 408 through the U-shaped microchannel. Four observation windows (see also Fig. 5 for their locations along the channel) are used: I, the entrance/exit region of the U-turn; II, the inlet branch of the U-turn that is closest to the magnet; III, the two corners of the U turn; IV, the channel outlet. The ferrofluid flow rate is $450 \mu \mathrm{l} / \mathrm{h}$. The block arrows indicate the ferrofluid flow and particle moving directions.

However, the two types of particles in the focused stream begin to be split when they travel into the outlet branch of the U-turn (window III). Due to their stronger magnetophoretic velocity, 15 $\mu \mathrm{m}$ particles migrate towards the lower wall of the outlet branch at a higher rate than $5 \mu \mathrm{m}$ particles. The displacement between these two particle streams continuously increases in window II and reaches the maximum (i.e., the best separation) when $15 \mu \mathrm{m}$ particles approaches the lower wall of the outlet branch in window I. The two separated particle streams then flow through different passages at the channel outlet in window IV. This observed particle focusingseparation process through the U-turn is captured reasonably by the numerical model in Fig. 4. We note that the particle separation distance is slightly affected by the $90^{\circ}$ turn at the outlet straight section, which should be removed or revised in future designs. An isometric view of the numerically predicted 3D particle trajectories for this separation is displayed in Fig. 5. As seen from the insets in the four observation windows I-IV, initially mixed and dispersed particles in the ferrofluid are first focused by negative magnetophoresis to a narrow stream flowing in the far 
corner of the bottom wall. They later undergo a continuous separation by size near the surface of the bottom wall.

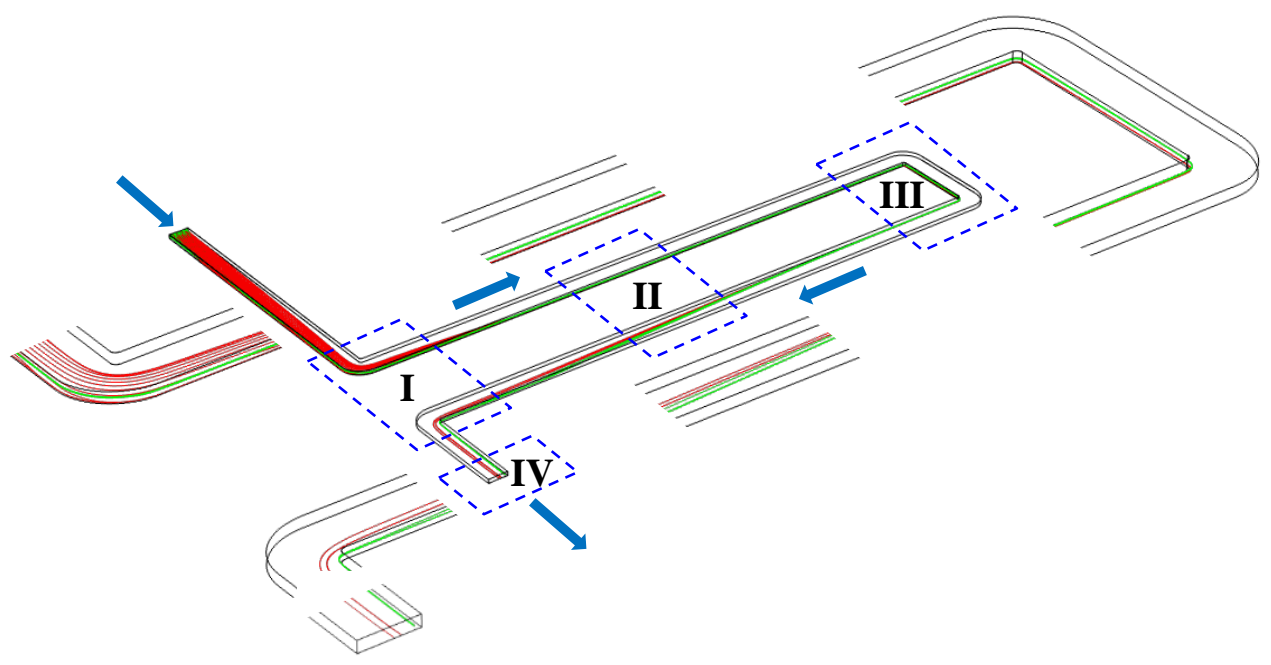

Fig. 5: Isometric view of the numerically predicted trajectories of $5 \mu \mathrm{m}$ (green lines) and $15 \mu \mathrm{m}$ (red lines) diamagnetic particles in $0.5 \times$ EMG 408 ferrofluid through the U-shaped microchannel at a volume flow rate of $450 \mu \mathrm{l} / \mathrm{h}$. The insets show the enlarged views of the particle trajectories in the same four observation windows as in Fig. 4. The block arrows indicate the ferrofluid flow and particle moving directions.

\subsection{Parametric effects on diamagnetic particle separation}

A quantitative analysis and comparison of experiment with simulation is presented below for the effects of ferrofluid flow rate, ferrofluid concentration, and magnet-channel distance on the diamagnetic particle separation in the U-shaped microchannel. Also presented are the numerically predicted channel geometry effects including the outlet-branch width and the gap between the inlet and outlet branches of the U-turn.

\section{A. Effect of ferrofluid flow rate}

Fig. 6(a1-a3) compares the experimental images and numerical predictions of $5 \mu \mathrm{m}$ and $15 \mu \mathrm{m}$ particles in $0.5 \times$ EMG 408 ferrofluid at the channel outlet under a volume flow rate of $450 \mu 1 / h$ 
(a1), $500 \mu \mathrm{l} / \mathrm{h}$ (a2) and $600 \mu \mathrm{l} / \mathrm{h}$ (a3), respectively. A quantitative comparison of the relative positions of the two separated particle streams between experiment (symbols) and simulation (lines) is presented in Fig. 6(b), where the relative particle positions are all determined with respect to the right sidewall of the outlet straight section in Fig. 6(a1-a3). Error bars are included for the experimental data points (symbols) to account for the spans of particle streams. A good agreement is obtained in between the experimental and modeling results. As seen from the trend of the two lines in Fig. 6(b) and as well the particle images in Fig. 6(a1-a3), the separation gap between the two particle streams grows with a decrease in the ferrofluid flow rate. It reaches the maximum at a flow rate of around $460 \mu \mathrm{l} / \mathrm{h}$, for which $15 \mu \mathrm{m}$ particles are able to reach the right sidewall of the microchannel. Further lowering the flow rate reduces the separation gap because $5 \mu \mathrm{m}$ particles still migrate towards the right sidewall while $15 \mu \mathrm{m}$ particles do not.

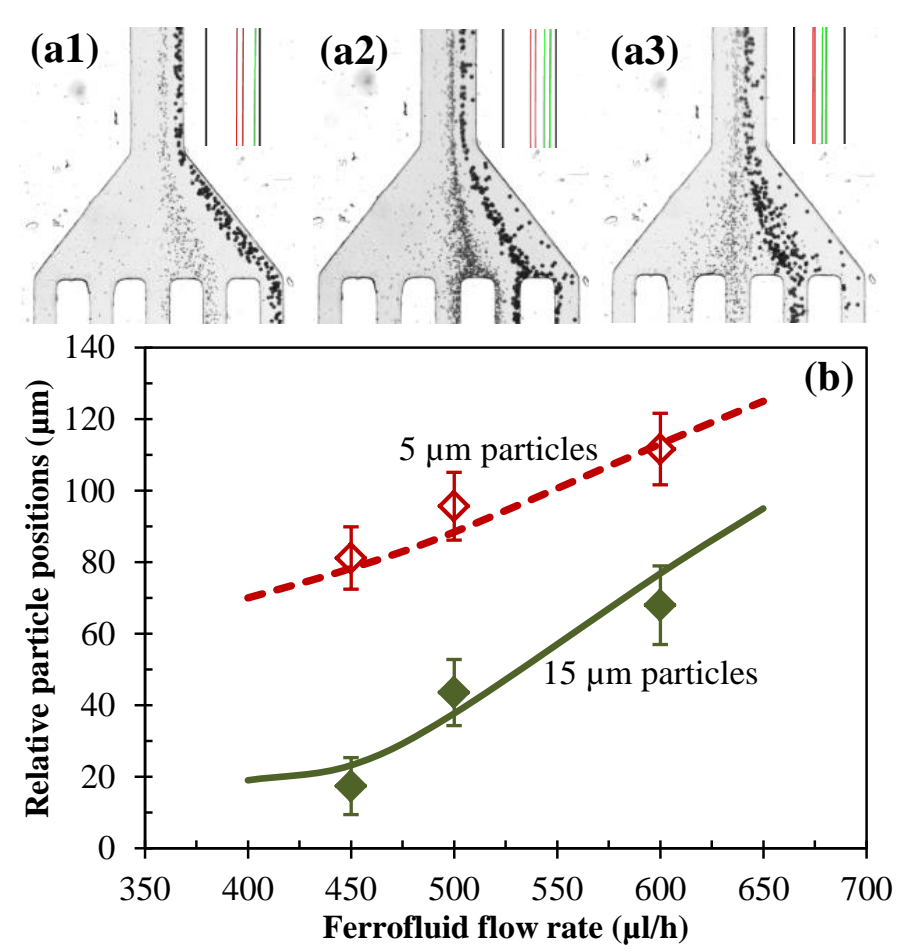

Fig. 6: Flow rate effects on diamagnetic particle separation in $0.5 \times$ EMG 408 ferrofluid through the U-shaped microchannel: (a1)-(a3) compare the experimentally obtained composite images 
with the numerically predicted particle trajectories (red lines for $5 \mu \mathrm{m}$ particles and green for 15

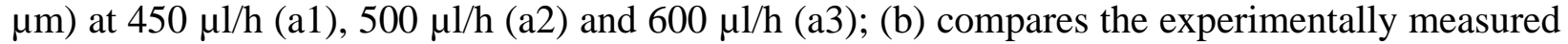
(symbols with error bars) and the numerically predicted (lines) particle positions at the end of the outlet straight section (with respect to the right sidewall).

\section{B. Effect of ferrofluid concentration}

Fig. 7 (a1-a3) shows the effect of ferrofluid concentration $(0.4 \times, 0.5 \times$ and $0.6 \times)$ on diamagnetic particle separation in the U-shaped microchannel at a fixed flow rate of $500 \mu \mathrm{l} / \mathrm{h}$. The separation gets worse when the ferrofluid concentration is varied from $0.5 \times$ to either $0.4 \times$ (i.e., decrease) or $0.6 \times$ (i.e., increase). This is because in $0.4 \times$ ferrofluid, $5 \mu \mathrm{m}$ particles are unable to receive a good magnetic focusing in the inlet-branch of the U-turn and hence still largely dispersed at the channel outlet causing an incomplete separation in Fig. 7(a1). In contrast, both $5 \mu \mathrm{m}$ and $15 \mu \mathrm{m}$ particles are well focused in $0.6 \times$ ferrofluid and able to reach the right sidewall of the outlet straight section thereby inhibiting the separation in Fig, 7(a3). This trend is quantitatively predicted by the numerical model as demonstrated by the comparison of the relative particle positions (with respect to the right sidewall of the outlet straight section) between experiment (symbols with error bars) and simulation (lines) in Fig. 7(b). Moreover, the simulation indicates that the best separation at $500 \mu \mathrm{l} / \mathrm{h}$ seems to take place in an approximately $0.53 \times$ ferrofluid. 

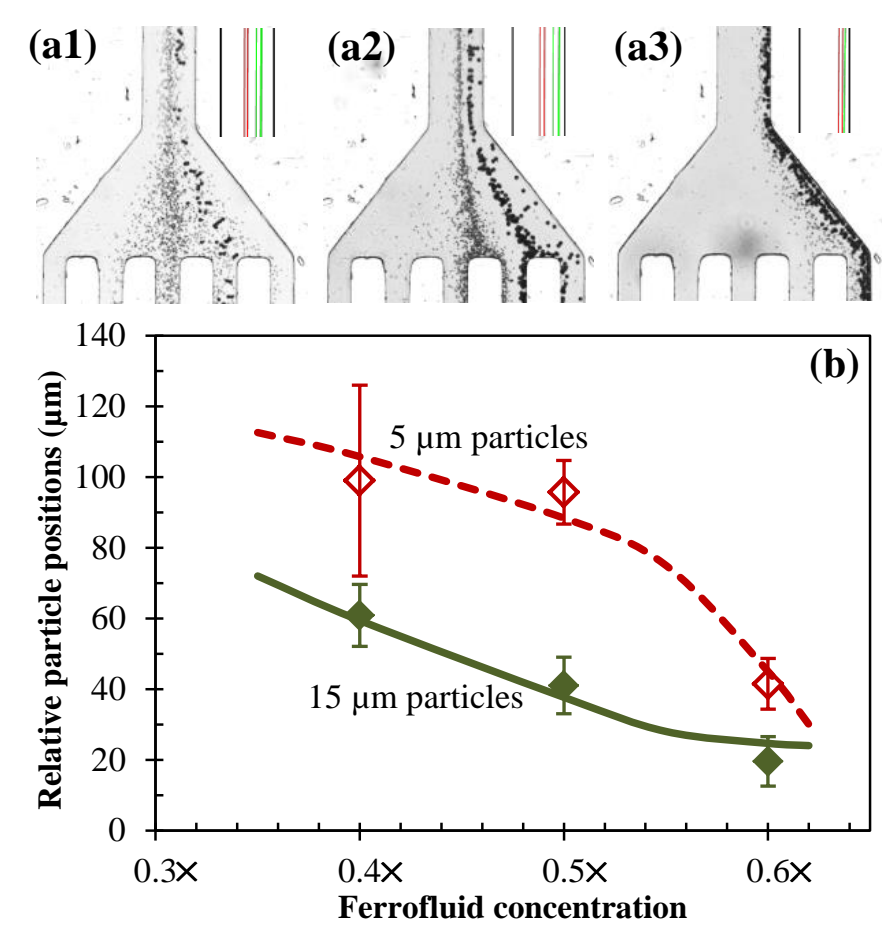

Fig. 7: Ferrofluid concentration effects on diamagnetic particle separation in the U-shaped microchannel: (a1)-(a3) compare the experimentally obtained composite images with the numerically predicted particle trajectories (red lines for $5 \mu \mathrm{m}$ particles and green for $15 \mu \mathrm{m}$ ) in 0.4× (a1), 0.5× (a2), and 0.6× (a3) EMG 408 ferrofluid; (b) compares the experimentally measured (symbols with error bars) and the numerically predicted (lines) particle positions at the end of the outlet straight section (with respect to the right sidewall). The ferrofluid flow rate is fixed at $500 \mu \mathrm{l} / \mathrm{h}$.

\section{Effect of magnet-channel distance}

Fig. 8(a1-a3) shows the effect of the distance between the magnet and the inlet branch of the Uturn, or simply the magnet-channel distance, on the separation images of $5 \mu \mathrm{m}$ and $15 \mu \mathrm{m}$ particles in $0.5 \times$ EMG 408 ferrofluid under a flow rate of $500 \mu \mathrm{l} / \mathrm{h}$. Fig. 8(b) compares the experimentally measured (symbols with error bars) and numerically predicted (lines) relative positions of the two particle streams with respect to the right sidewall of the outlet straight section. With an increase in the magnet-channel distance from $600 \mu \mathrm{m}$ (a1) to $800 \mu \mathrm{m}$ (a2) and $1000 \mu \mathrm{m}(\mathrm{a} 3)$, the separation gap between the two particle streams increases as seen from the numerical lines in Fig. 8(b). However, the best separation seems to take place at the $800 \mu \mathrm{m}$ 
magnet-channel distance from both the experiment and simulation. It is because neither type of particles receives a good magnetic focusing at the $1000-\mu \mathrm{m}$ magnet-channel distance, which yields a considerable span for each particle stream as viewed in Fig. 8(a3). At the distance of 600 $\mu \mathrm{m}$, both types of particles are fully deflected with a tight focusing and hence overlap with each other at near the right sidewall of the outlet straight section. Overall, the numerical model predicts the experimental results with a reasonable agreement.

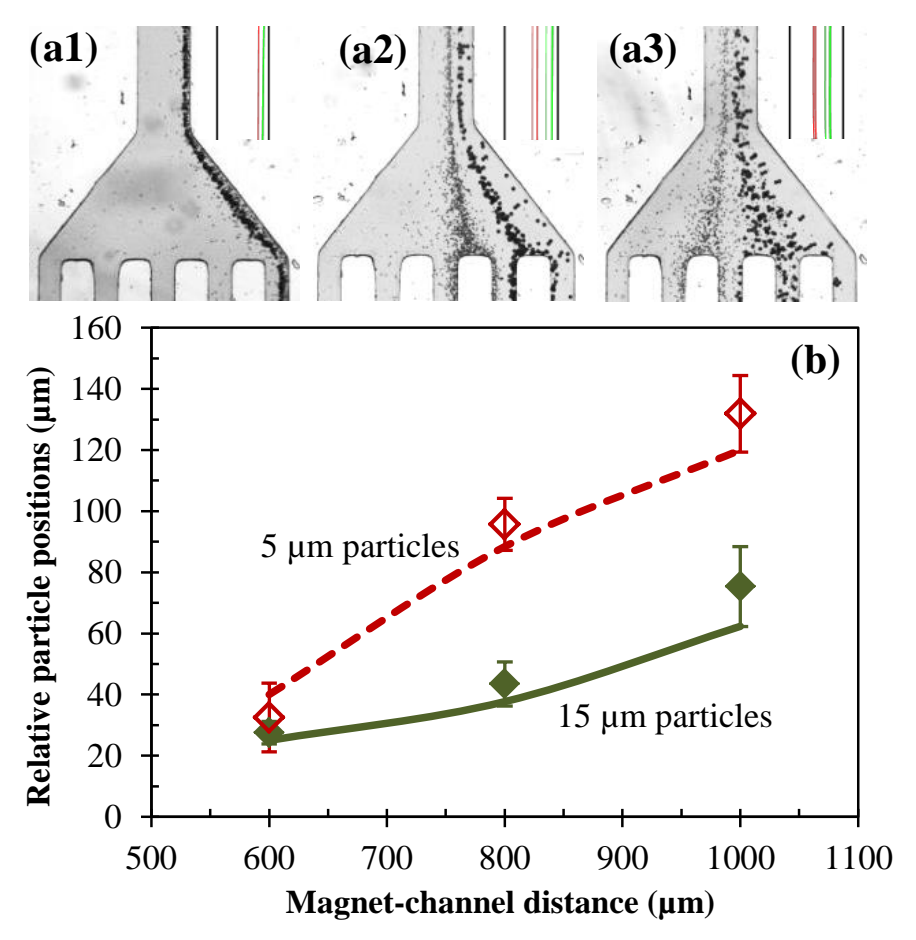

Fig. 8: Magnet-channel distance effects on diamagnetic particle separation in 0.5× EMG 408 ferrofluid through the U-shaped microchannel: (a1)-(a3) compare the experimentally obtained composite images with the numerically predicted particle trajectories (red lines for $5 \mu \mathrm{m}$ particles and green for $15 \mu \mathrm{m}$ ) when the permanent magnet is $600 \mu \mathrm{m}$ (a1), $800 \mu \mathrm{m}$ (a2), and $1000 \mu \mathrm{m}$ (a3) away from the inlet branch of the U-turn; (b) compares the experimentally measured (symbols with error bars) and the numerically predicted (lines) particle positions at the end of the outlet straight section (with respect to the right sidewall). The ferrofluid flow rate is fixed at $500 \mu \mathrm{l} / \mathrm{h}$.

\section{Effect of outlet-branch width}

The above parametric studies have validated our 3D numerical model. We therefore use this model to further examine the effects of two other geometrical parameters that are not tested 
experimentally. Fig. 9(a1-a3) shows the width effect of the outlet branch of the U-turn on the numerically predicted trajectories of $5 \mu \mathrm{m}$ and $15 \mu \mathrm{m}$ particles in $0.5 \times$ EMG 408 ferrofluid at the entrance/exit region of the U-turn (i.e., window I in Fig. 4 and Fig. 5). Other parameters are fixed and referred to the experiment in Fig. 7. Fig. 9(b) compares the predicted relative (center) positions of the two particle streams with respect to the right sidewall at the outlet straight section. As the trend shows, the gap between the two streams increases with an increase in the outlet-branch width, which is also visible from the particle trajectories in Fig. 9(a1-a3). This enhanced separation arises from the slower flow velocity in a wider channel, where both types of particles will have a longer retention time to be magnetically deflected in the ferrofluid. However, as viewed from the predicted trajectories in Fig. 9(a1-a3) and as well the error bars in Fig. 9(b), the two particle streams both expand with an increase in the outlet-branch width, which should be taken into account in optimizing the U-shaped microchannel for diamagnetic particle separation.

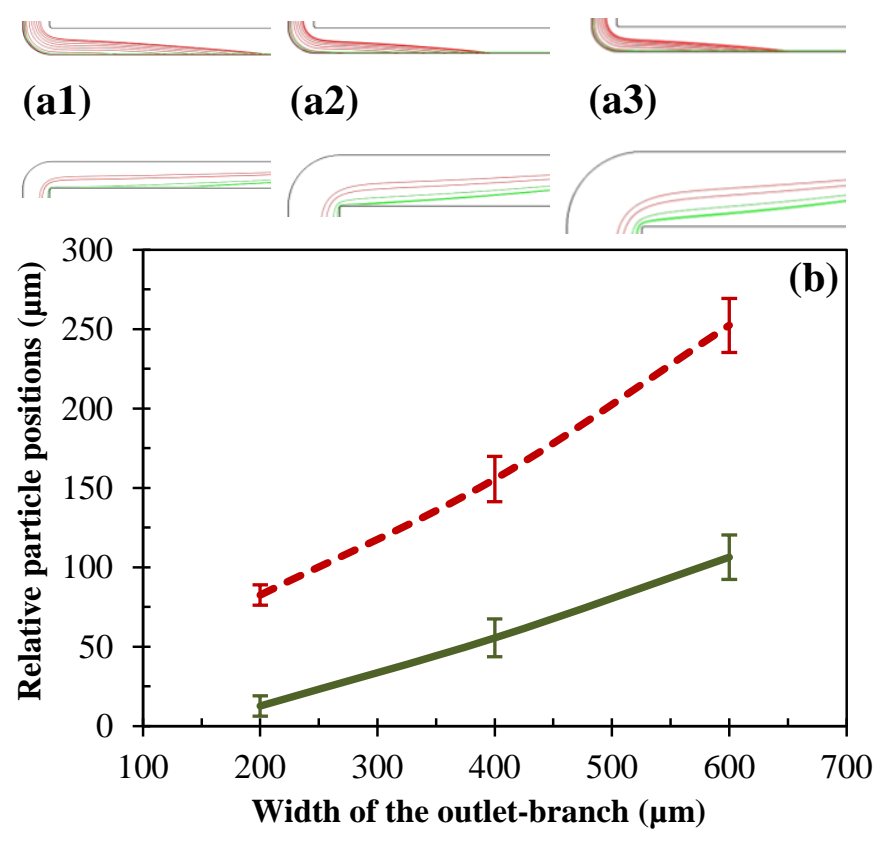

Fig. 9: Outlet-branch width effects on diamagnetic particle separation in $0.5 \times$ EMG 408 ferrofluid through the U-shaped microchannel: (a1)-(a3) show the numerically predicted 
trajectories of $5 \mu \mathrm{m}$ (red lines) and $15 \mu \mathrm{m}$ (green lines) particles for an outlet-branch of $200 \mu \mathrm{m}$ (a1), $400 \mu \mathrm{m}$ (a2), and $600 \mu \mathrm{m}$ (a3) wide; (b) shows the numerically predicted particle positions (error bars included to cover the span of the particle streams in a1, a2 and a3) at the end of the outlet straight section (with respect to the right sidewall). Other parameters are referred to Fig. 7.

\section{E. Effect of the gap width between inlet and outlet branches}

Fig. 10(a1-a3) shows the effect of the gap width between the inlet and outlet branches of the Uturn on the numerically predicted trajectories of $5 \mu \mathrm{m}$ and $15 \mu \mathrm{m}$ particles. Fig. 10(b) shows the predicted edge-to-edge separation distance of the two particle streams at the end of the outlet straight section when the branch-gap width is varied from $600 \mu \mathrm{m}$ to $1000 \mu \mathrm{m}$. Under the tested conditions, the particle separation is not a linear function of the branch-gap width and seems to achieve the best with a gap width of $830 \mu \mathrm{m}$. At the $600 \mu \mathrm{m}$ branch-gap width, $5 \mu \mathrm{m}$ and $15 \mu \mathrm{m}$ particles can both have a nearly full-width deflection and are hence barely separated as seen from Fig. 10(a1). At the $1000 \mu \mathrm{m}$ branch-gap width, both types of particles still try to approach the sidewall at the exit of the U-turn and also experience a greater dispersion in the process, which yields a reduced separation as demonstrated in Fig. 10(a3).

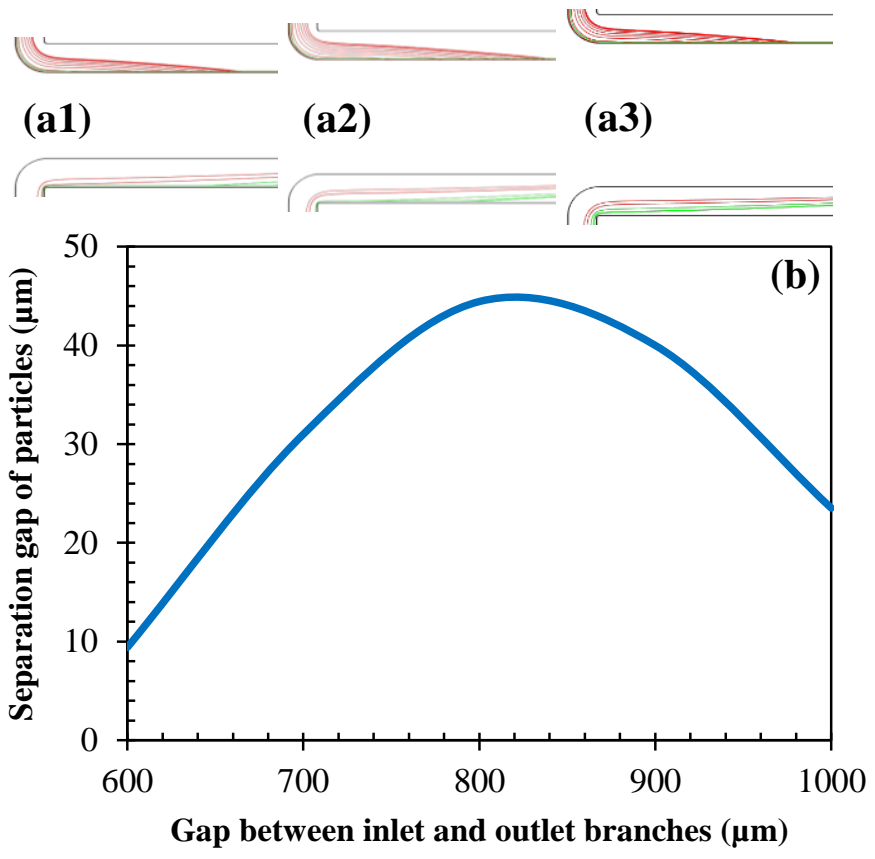


Fig. 10: The effect of the gap width between inlet and outlet branches of the U-turn on diamagnetic particle separation in $0.5 \times$ EMG 408 ferrofluid through the U-shaped microchannel: (a1)-(a3) show the numerically predicted trajectories of $5 \mu \mathrm{m}$ (red lines) and $15 \mu \mathrm{m}$ (green lines) particles for a gap of $600 \mu \mathrm{m}$ (a1), $800 \mu \mathrm{m}$ (a2), and $1000 \mu \mathrm{m}$ (a3); (b) shows the numerically predicted separation distance between the two particle streams at the end of the outlet straight section. Other parameters are referred to Fig. 7.

\section{Conclusions}

We have demonstrated a continuous-flow sheathless diamagnetic particle separation in ferrofluids through U-shaped microchannels. Due to the action of a size-dependent magnetic force, diamagnetic particles are focused into a single stream in the inlet branch of the U-turn and then continuously separated into two streams in its outlet branch. The parametric effects of ferrofluid flow rate, ferrofluid concentration, and magnet-channel distance on this diamagnetic particle separation are experimentally studied. We have also developed a 3D numerical model to simulate the particle trajectories in ferrofluid flows through the U-shaped microchannel under the experimental conditions. The numerical predictions are found to agree well with the experimental observations. Moreover, we have used the validated 3D numerical model to investigate the geometric effects of the U-turn, including the width of the outlet branch and the gap width between the inlet and outlet branches, on diamagnetic particle separation in ferrofluids. Particularly interesting is the finding that increasing the outlet-branch width of the U-turn can significantly enhance the particle separation. The obtained experimental and numerical results in this work will aid in the optimal design and control of future U-shaped microchannels for the best separation.

\section{Acknowledgments}


This work was partially supported by NSF under grant CBET-1150670 (XX) and by 111 Project under grant B12019 (LY).

\section{Appendix}

We prove below that the ferrofluid concentration gradient induced by a non-uniform magnetic field has an insignificant influence on the trajectory of diamagnetic particles in the U-shaped microchannel. To save computational time, we have developed a $2 \mathrm{D}$ numerical model to consider the coupling between ferrofluid flow and concentration fields. This full model has also been compared with a 2D numerical model that ignores the ferrofluid concentration gradient and uses the same equations as in the main text. In the former full numerical model, the ferrofluid flow field is solved from transient Navier-Stokes equations,

$$
\rho\left(\frac{\partial \mathbf{u}}{\partial t}+\mathbf{u} \cdot \nabla \mathbf{u}\right)=-\nabla p+\eta \nabla^{2} \mathbf{u}+\mathbf{f}_{m}
$$

where the magnetic body force, $\mathbf{f}_{m}$ as defined in Eq. (3) of the main text, is a function of ferrofluid concentration, $c$, due to the concentration dependence of ferrofluid magnetization, $\mathbf{M}_{f}$,

$$
\mathbf{M}_{f}=c \mathbf{M}_{d}[\operatorname{coth}(\alpha)-1 / \alpha]
$$

The ferrofluid concentration field is solved from the convection-diffusion equation,

$$
\frac{\partial c}{\partial t}+\nabla \cdot\left(-D \nabla c+\mathbf{u c}+\mathbf{u}_{n p} \mathrm{c}\right)=0
$$

where $\mathbf{u}_{n p}$ is the magnetophoretic velocity of ferrofluid nanoparticles (which are superparamagnetic),

$$
\mathbf{u}_{n p}=\frac{\mu_{0} d^{2}[\operatorname{coth}(\alpha)-1 / \alpha] \mathbf{M}_{d} \cdot \nabla \mathbf{H}}{18 \eta}
$$

Eq. (A1) and Eq. (A3) along with Eq. (1) in the main text are solved together in COMSOL 4.4 using a time-dependent solver. A normal inflow velocity $(15.6 \mathrm{~mm} / \mathrm{s}$, corresponding to a volume 
flow rate of $450 \mu \mathrm{l} / \mathrm{h})$ and a uniform ferrofluid concentration $\left(c=c_{0}=0.5 \times 1.2 \%\right.$ for $0.5 \times$ EMG 408 ferrofluid) are applied to the inlet of the U-shaped microchannel. The outlet is at zero pressure and has a zero concentration gradient. All walls of the microchannel are assumed noslip and no-penetrating.

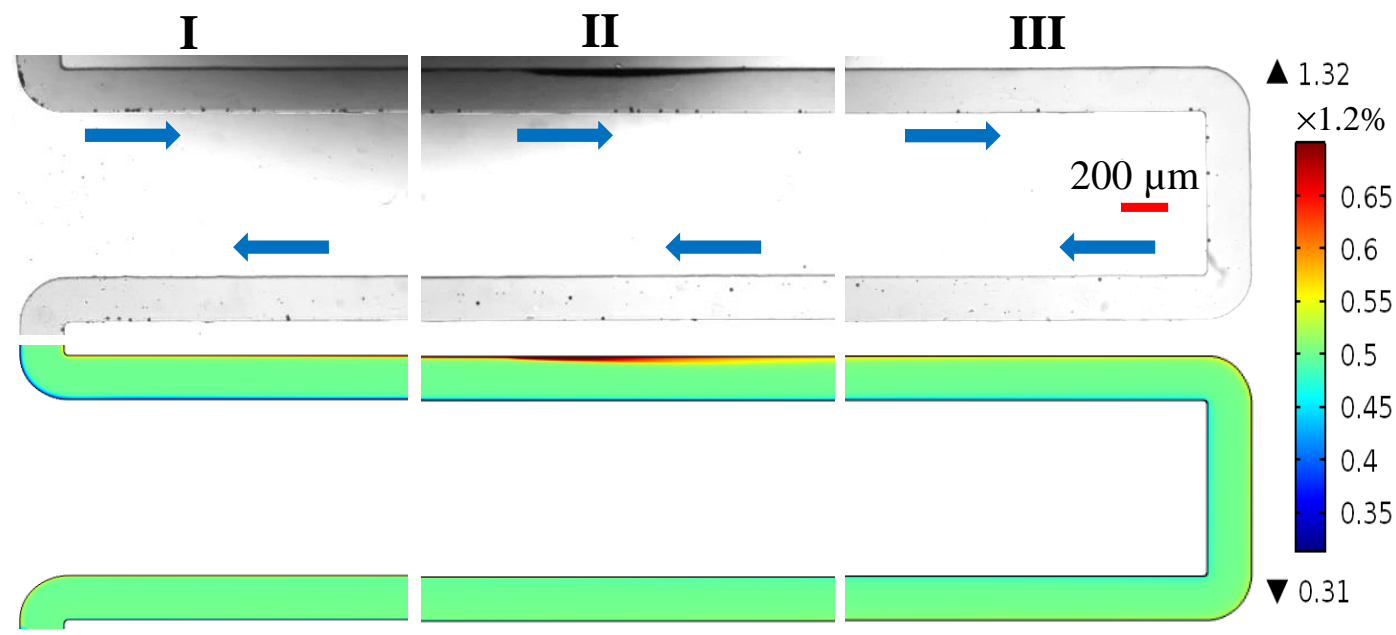

Fig. A1: Comparison of the experimentally recorded snapshot images (top row) and the numerically predicted concentration fields (bottom row) of ferrofluid at three observation windows of the U-shaped microchannel (see Fig. 5 of the main text for their locations). All experimental conditions are identical to those listed in the caption of Fig. 4. The block arrows indicate the ferrofluid flow and particle moving directions.

Fig. A1 shows the comparison of ferrofluid concentration between experimental images and numerical predictions (from the 2D full model) at the three observation windows I, II and III of the U-shaped microchannel. As they experience positive magnetophoresis, ferrofluid nanoparticles migrate towards the region of the highest magnetic field strength. This causes an increased ferrofluid concentration on the wall of the inlet branch that is nearest to the magnet in Window II, which is qualitatively predicted by the 2D full model in Fig. A1. Meanwhile, there occurs a slight depletion of ferrofluid on the walls that are away from the magnet. However, the ferrofluid concentration in the bulk region of the entire channel remains almost unvaried at the initial concentration. Therefore, the trajectories of diamagnetic particles in the ferrofluid are not 
expected to be significantly affected by the magnet-induced ferrofluid concentration gradient. This is verified by comparing the predicted particle trajectories from $2 \mathrm{D}$ numerical models with and without consideration of the coupling between ferrofluid flow and concentration fields. As seen from Fig. A2, there is no visible difference between the two results.

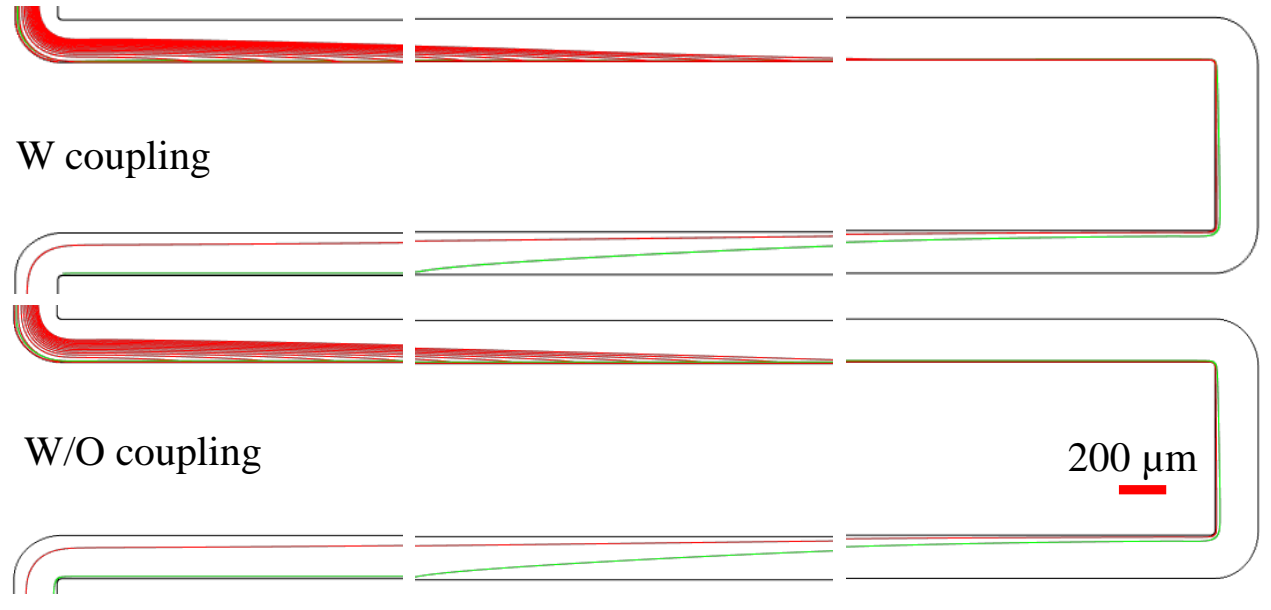

Fig. A2: Comparison of the predicted trajectories of $5 \mu \mathrm{m}$ (red lines) and $15 \mu \mathrm{m}$ (green lines) diamagnetic particles from 2D numerical models with (top row) and without (bottom row) consideration of the coupling between ferrofluid flow and concentration fields, respectively, in $0.5 \times$ EMG 408 through the U-shaped microchannel. All working conditions remain identical between the two models.

\section{References}

[1] N. Pamme, Lab Chip 7 (2007) 1644-1659.

[2] A. Lenshof, T. Laurell, Chem. Soc. Rev. 39 (2010) 1203-1217.

[3] Y. Gao, W. Li and D. Pappas, Analyst 138 (2013) 4714-4721.

[4] C. W. Shields IV, C. D. Reyes and G. P. Lopez, Lab Chip 15 (2015) 1230-1249.

[5] T. Laurell, F. Petersson, and A. Nilsson, Chem. Soc. Rev. 36 (2007) 492-506.

[6] S. C. Lin, X. Mao, T. J. Huang, Lab Chip 12 (2012) 2766-2770.

[7] R. Pethig, Biomicrofluid. 4 (2010) 022811.

[8] B. Çetin, D. Li, Electrophoresis 32 (2011) 2410-2427. 
[9] T. Z. Jubery, S. K. Srivastava, P. Dutta, Electrophoresis 35 (2014) 691-713.

[10] H. Tsutsui, C. M. Ho, Mech. Res. Comm. 35 (2009) 92-103.

[11] J. M. Martel, M. Toner, Annu. Rev. Biomed. Eng. 16 (2014) 371-396.

[12] H. Amini, W. Lee, D. Di Carlo, Lab Chip 14 (2014) 2739-2761.

[13] N. Pamme, Lab Chip 6 (2006) 24-38.

[14] R. Yang, H. Hou, Y. Wang, L. Fu, Sens. Act. B 224 (2016) 1-15.

[15] M. Hejazian, W. H. Li, N.-T. Nguyen, Lab Chip 15 (2015) 959-970.

[16] S. H. Cho, J. M. Godin, C. H. Chen, W. Qiao, H. Lee, and Y. H. Lo, Biomicrofluid. 4 (2010) 043001.

[17] A. Kayani, K. Khoshmanesh, S. A. Ward, A. Mitchell, and K. Kalantar-Zadeh, Biomicrofluid. 6 (2012) 031501.

[18] D. R. Gossett, W. M. Weaver, A. J. Mach, S. C. Hur, H. T. Tse, W. Lee, H. Amini, D. Di Carlo, Anal. Bioanal. Chem. 397 (2010) 3249-3267.

[19] H. Watarai, Annu. Rev. Anal. Chem. 6 (2013) 353-378.

[20] Karimi, S. Yazai, A.M. Ardekani, Biomicrofluid. 7 (2013) 021501.

[21] H. Watarai, M. Suwa, and Y. Iiguni, Anal. Bioanal. Chem. 378 (2004) 1693-1699.

[22] M. A. M. Gijs, F. Lacharme, U. Lehmann, Chem. Rev. 110 (2010) 1518-1563.

[23] N. T. Nguyen, Microfluid. Nanofluid. 12 (2012) 1-16.

[24] Q. Cao, X. Han, L. Li, Lab Chip 14 (2014) 2762-2777.

[25] M. Zborowski, L. Sun, L. R. Moore, P. S. Williams, J. J. Chalmers, J. Magnet. Magnet. Mater. 194 (1999) 224-230.

[26] N. Pamme, J. C. T. Eijkel, A. Manz, J. Magnet. Magnet. Mater. 307 (2006) 237-244. 
[27] M. D. Tarn, S. A. Peyman, D. Robert, A. Iles, C. Wilhelm, N. Pamme, J. Magnet. Magnet. Mater. 321 (2009) 4115-4122.

[28] M. Hoyos, L. Moore, P. S. Williams, M. Zborowski, J. Magnet. Magnet. Mater. 323 (2011) 1384-1388.

[29] J. H. Kang, S. Krause, H. Tobin, A. Mammoto, M. Kanapathipillai, D. E. Ingber, Lab Chip 12 (2012) 2175-2181.

[30] S. Kim, S. I. Han, M. J. Park, C. W. Jeon, Y. D. Joo, I. H. Choi, K. H. Han, Anal. Chem. 86 (2013) 2779-2786.

[31] P. Chen, Y. Huang, K. Hoshino, X. J. Zhang, Sci. Rep. 5 (2015) 8745, doi:10.1038/srep08745.

[32] C. Chang, W. Huang, S. I. Jalal, B. Chan, A. Mahmood, S. Shahda, B. H. O'Neil, D. E. Matei and C. A. Savran, Lab Chip 15 (2015) 1677-1688.

[33] Winkleman, R. Perez-Castillejos, K. L. Gudiksen, S. T. Phillips, M. Prentiss, G. M. Whitesides, Anal. Chem. 79 (2007) 6542-6550.

[34] J. H. Kang, S. Choi, W. Lee, J. K. Park, J. Am. Chem. Soc. 130 (2007) 396-397.

[35] S.A. Peyman, E.Y. Kwan, O. Margarson, A. Iles, N. Pamme, J. Chromatogr. A 1216 (2009) 9055-9062.

[36] Y. K. Hahna, J. K. Park, Lab Chip 11 (2011) 2045-2048.

[37] T. Zhu, F. Marrero, L. Mao, Microfluid. Nanofluid. 9 (2010) 1003-1009.

[38] A.R. Kose, B. Fischer, L. Mao, H. Koser, Proc. Natl. Acad. Sci. 106 (2009) 21478-21483.

[39] S. Bucak, S. Sharpe, S. Kuhn, T. A. Hatton, Biotechnol. Prog. 27 (2011) 744-750

[40] W. Zhao, T. Zhu, R. Cheng, Y. Liu, J. He, H. Qiu, L. Wang, T. Nagy, T. D. Querec, E. R. Unger, L. Mao, Adv. Fun. Mat. 2015, in press. DOI: 10.1002/adfm.201503838. 
[41] M. Vojtisek, M.D. Tarn, N. Hirota, N. Pamme, Microfluid. Nanofluid. 13 (2012) 625-635.

[42] S. Shen, H. Hwang, Y. K. Hahn, J. K. Park, Anal. Chem. 84 (2012) 3075-3081.

[43] T. Zhu, R. Cheng, S.A. Lee, E. Rajaraman, M.A. Eiteman, T.D. Querec, E.R. Unger, L. Mao, Microfluid. Nanofluid. 13 (2012) 645-654.

[44] L. Liang, C. Zhang, X. Xuan, X., Appl. Phys. Lett. 102 (2013) 234101.

[45] T. Zhu, R. Cheng, Y. Liu, J. He, L. Mao, Microfluid. Nanofluid. 17 (2014) 973-982.

[46] K. A. Mirica, S. S. Shevkoplyas, S. T. Phillips, M. Gupta, G. M. Whitesides, J. Am. Chem. Soc. 131 (2009) 10049-10058.

[47] R. E. Rosensweig, Annu. Rev. Fluid Mech. 19 (1987) 437-461.

[48] T. Zhu, R. Cheng, L. Mao, Microfluid. Nanofluid. 11 (2011) 695-701.

[49] L. Liang, J. Zhu, X. Xuan, Biomicrofluid. 5 (2011) 034110.

[50] J. Zeng, C. Chen, P. Vedantam, V. Brown, T. R. J. Tzeng, X. Xuan, J. Micromech. Microeng. 22 (2012) 105018.

[51] L. Liang, X. Xuan, Microfluid. Nanofluid. 13 (2012) 637-643.

[52] J. J. Wilbanks, G. Kiessling, J. Zeng, C. Zhang, X. Xuan, J. Appl. Phys. 115 (2014) 044907.

[53] G. P. Zhu, M. Hejiazan, X. Y. Huang, N. T. Nguyen, Lab Chip 14 (2014) 4609-4615.

[54] M. Hejazian, N. T. Nguyen, Lab Chip 15 (2015) 2998-3005.

[55] J. Zeng, Y. Deng, P. Vedantam, T. R. Tzeng, and X. Xuan, J. Magnet. Magnet. Mater. 346 (2013) 118-123.

[56] L. Liang, X. Xuan, Biomicrofluid. 6 (2012) 044106.

[57] M. Benelmekki, L. M. Martinez, J. S. Andreu, J. Camachod, J. Faraudo, Soft Matt. 8 (2012) 6039-6047.

[58] J. Zhu, L. Liang, X. Xuan, Microfluid. Nanofluid. 12 (2012) 65-73. 
[59] Y. Zhou, D. T. Kumar, X. Lu, A. Kale, J. DuBose, Y. Song, J. Wang, D. Li, X. Xuan, Biomicrofluid. 9 (2015) 044102.

[60] T. Zhu, D. J. Lichlyter, M. A. Haidekker, L. Mao, Microfluid. Nanofluid. 10 (2011) 1233 1245.

[61] R. Cheng, T. Zhu, L. Mao. Microfluid. Nanofluid. 16 (2014) 1143-1154.

[62] G. Zhu, N. T. Nguyen, Microfluid. Nanofluid. 13 (2012) 655-663.

[63] C. Y. Wen, K. P. Liang, H. Chen, L. M. Fu, Electrophoresis 32 (2011) 3268-3276.

[64] E. P. Furlani, Permanent magnet and electromechanical devices: materials, analysis, and applications 2001, Academic Press, New York.

[65] X. Han, Y. Feng, Q. Cao, L. Li, Microfluid. Nanofluid. 18 (2015) 1209-1220. 